Title: Managing expense and expectation in a treatment revolution: Problematizing prioritisation through an exploration of hepatitis C treatment 'benefit'.

Author name and affiliations: Magdalena Harris

London School of Hygiene and Tropical Medicine, 15-17 Tavistock Place, London WC1H9SH, UK.

Corresponding author: Magdalena Harris, London School of Hygiene and Tropical Medicine, 15-17 Tavistock Place, London WC1H9SH, UK.

magdalena.harris@lshtm.ac.uk ,+44 7519619865. 


\section{Managing expense and expectation in a treatment revolution: Problematizing prioritisation through an exploration of hepatitis C treatment 'benefit'.}

\section{ABSTRACT}

Background: Direct-acting antivirals (DAAs) have transformed the hepatitis C (HCV) treatment landscape. These highly effective drugs are, however, not available to all. In a context of DAA rationing, clinicians are advised to "manage patient expectations" about the benefits of a HCV cure. This directive particularly pertains to people with minimal liver damage and those who have ceased injecting: populations negated in contemporary prioritisation debates.

Methods: This paper engages with the assumptions underpinning HCV treatment prioritisation discourses to explore the concept of treatment 'benefit' from patient perspectives. Data are from a qualitative longitudinal study exploring treatment transitions and decision-making from 2012-2015. Participants comprised 28 people living with HCV, ten treatment providers and eight stakeholders, based in London, United Kingdom (UK). One hundred hours of clinic observations were conducted at two HCV treatment hospitals. Thematic analyses pertaining to treatment expectation and outcome inform this paper.

Findings: Twenty-two participants commenced treatment. The majority who were unable to access DAAs chose to commence interferon-based treatment immediately rather than wait. Participants accounted for treatment urgency in relation to three interrelated narratives of hope and expectation. HCV treatment promised: social reconnection; social redemption and a return to 'normality'. For many with successful treatment outcomes, these benefits appeared to be realised. Conclusion: The DAA era heralds a discursive shift: from 'managing [interferon] risk and difficulty' to 'managing [DAA] expense and expectation'. Calls to 'manage patient expectations' about the benefits of HCV cure are predicated on clinical benefits only, negating the social impacts of living with HCV. The public health priorities commonly articulated in treatment prioritisation debates are not consistent with those of people managing illness in their daily lives. During this 'treatment revolution' there is a need to be cognisant of the multiple publics living with the virus and the treatment needs of those who do not fit population-health scenarios.

Keywords: hepatitis C; treatment benefit; prioritisation; rationing; patient expectations; qualitative research. 